\title{
E1 migrante climático y su reconocimiento en el ordenamiento jurídico internacional
}

\section{Haydée Bermúdez Guevara ${ }^{1, *}$}

${ }^{1}$ Centro del Agua del Trópico Húmedo para América Latina y el Caribe (CATHALAC), República de Panamá.

*Autor para correspondencia. Correo electrónico: bermudez@usal.es

Recibido: 15 de marzo de 2017

Aceptado: 11 de abril de 2017

\begin{abstract}
.
Climate change is one of the most controversial topics because of its political and economic implications. Accordingly, an important debate has arisen related to the need to legally recognize a new category of migrants known as environmental refugees or migrants. There is currently, a well-known refusal of governments to recognize an additional refugee category from the ones already covered by the 1951 Refugee Convention. There is also a lack of consensus even in the scientific and legal community. This phenomenon should not be seen as an argument from developing countries but as a topic that must be addressed by all countries, as a decision making mechanism prior to a well-founded political and legal decision.
\end{abstract}

Keywords. Climate change; Climate migrant; Legal recognition.

\section{Resumen.}

El cambio climático es uno de los temas más controvertidos por las implicaciones políticas y económicas que tiene. De igual manera, nace el debate sobre la necesidad de reconocer legalmente una nueva categoría de migrantes conocidos como refugiados o migrantes ambientales. Actualmente, es amplia la negativa de los gobiernos a reconocer una categoría adicional a los refugiados ya comprendidos en la Convención de Refugiados de 1951. Esto, aunado a la falta de consenso inclusive en la comunidad científico y legal. Este fenómeno no debe ser visto como un argumento de los países en vías de desarrollo sino como un tema que debe ser abordado por todos los países, como mecanismo previo a una toma de decisión política y legal, bien fundamentada.

Palabras clave. Cambio climático; Migrante climático; Reconocimiento jurídico.

\section{Introducción.}

Los efectos del cambio climático son aceptados ampliamente por la comunidad científica, y actualmente también por la comunidad política mundial. Dentro de este proceso, se busca que las Conferencias de las Partes (COP) de la Convención Marco de las Naciones Unidas sobre el Cambio Climático sea el foro ideal donde la diplomacia climática muestre sus mejores resultados. Hasta el momento, 
Invest. pens. crit. (ISSN 1812-3864)

Vol. 5, No. 1, enero-abril 2017

pp. 69-76

las COP's han servido para resaltar la importancia del vínculo entre ciencia y políticas públicas, y para que los gobiernos nacionales, regionales y locales asuman su rol de actores políticos necesarios.

Es en este contexto, que se presenta la necesidad de regular una situación de hecho que afecta a un grupo cada vez más amplio de personas que se ven impactados por los efectos del cambio climático o por eventos ambientales extremos, ellos son los migrantes climáticos. ${ }^{1}$

ACNUR estima que para el 2050, existirán de 250 a 1000 desplazados ambientales; no obstante, el cálculo más aceptado es el del profesor Norman Myers de la Universidad de Oxford, quien ha pronosticado una migración de 200 millones de personas para el año 2050 producto de fenómenos relacionados con el cambio climático [1]. Por otro lado, Egea y otros indican que son muchos los esfuerzos por cuantificar esta tipología de migrantes pero que ese "baile de cifras" a veces tienen un carácter alarmista [2].

Si bien las cifras no son precisas, es importante resaltar que hasta los números más conservadores requerirán de acciones concretas y urgentes por parte de la comunidad política internacional, pues el no querer regular una situación de hecho, impactará a corto, mediano y largo plazo, tanto en países en vías de desarrollo como a los desarrollados. Unos porque serán emisores y otros receptores de amplios flujos migratorios.

Razón por la cual se hace más patente la necesidad de estudios con base científica, que sirvan de guía a los tomadores de decisiones en la elaboración de sus políticas públicas, partiendo de un reconocimiento formal del migrante climático. De hecho, Brown [1] hace hincapié en este sentido, e indica que es necesario avanzar en estudios con sólidas bases científicas que puedan provocar estos cambios políticos y legislativos.

El presente trabajo pretende contribuir al debate haciendo hincapié sobre esta correlación, y tomando como casos de estudio algunos proyectos relevantes o inclusive el fallo que niega el estatus jurídico al primer solicitante de refugio climático.

\section{$2 \quad$ El concepto de migrante ambiental}

El término refugiado o migrante ambiental fue utilizado por primera vez en 1976 por el ambientalista Lester Russel Brown, fundador de Worldwatch Institute y del Earth Policy Institute, para referirse a una categoría de migrantes por causas ambientales, y hasta ese momento no habían sido considerados

\footnotetext{
${ }^{1}$ Existe un sin numero de términos para referirse al migrante por causas ambientales: refugiado climático, migrante ambiental, desplazados ambientales, entre otros.
} 
Invest. pens. crit. (ISSN 1812-3864)

Vol. 5, No. 1, enero-abril 2017

pp. 69-76

y se encontraban en un limbo jurídico.

Es más, en la $94^{a}$ sesión de la OIM, se discutió la relación de las migraciones y el ambiente, y se propuso la siguiente definición de migrantes ambientales como "las personas o grupos de personas que por razones imperiosas de cambios repentinos o progresivos en el medio ambiente, que afecten negativamente su vida o sus condiciones, se ven obligados a abandonar su lugar de residencia habitual o deciden hacerlo ya sea temporal o permanentemente, ya sea en su país o en el extranjero” [3]).

Desde entonces y hasta la fecha, no son muchos los avances en la materia. De hecho no se ha llegado a un consenso para definir quién es este migrante climático, y si debe existir una reglamentación especial que lo proteja.

Para autores como Gray, Egea o Soledad, el tema ha sido visto de una manera alarmista alegándose que existen causas ambientales para las migraciones, cuando éstas son más bien de carácter económico, social o político. Aunado a ello, desestiman la migración ambiental como efecto de eventos climáticos extremos e indican que en esos casos, la migración es interna y temporal. Inclusive a consideración de estos autores, el tema ha sido politizado, existiendo la posibilidad posteriormente de ser utilizado como motivo de reforma para una normativa migratoria más estricta [4].

Si bien debemos admitir la complejidad existente para encontrar las causas de ese tipo de migración; no por ello, debemos desconocer la necesidad de una protección especial del migrante climático. Más bien considero que se deben ampliar los estudios al respecto pues una causa económica puede ser el resultado de causas ambientales que afectan los principales medios de producción de un pueblo determinado.

Para la Organización Internacional para las Migraciones (OIM), migración es "el movimiento de personas en el que se observa la coacción, incluyendo la amenaza a la vida y su subsistencia, bien sea por causas naturales o humanas, dando como ejemplo el movimiento de refugiados y de desplazados internos, así como personas desplazadas por desastres naturales o ambientales, desastres nucleares o químicos, hambruna o proyectos de desarrollo"[3]. En esta última definición, vemos que ya se incorporan causales ambientales, si bien no es aceptado internacionalmente como una causa de la migración existente.

Aun así, es interesante analizar las conclusiones del proyecto financiado por la Comisión Europea denominado EACH FOR (Cambio Medioambiental y Migración Forzada), destinado a sistematizar 
Invest. pens. crit. (ISSN 1812-3864)

Vol. 5, No. 1, enero-abril 2017

pp. $69-76$

diversas experiencias donde las adversidades medioambiental han afectado la migración. A partir de

los 23 casos analizados, se desarrolló una metodología con la que se analizaron las posibles causas directas e indirectas a estos desplazamientos.

De éstas, cuatro investigaciones se realizaron en América Latina, a saber: en la isla la Española (Haití y República Dominicana), México, Ecuador y Argentina. ${ }^{2}$ La investigación en Ecuador arrojo resultados interesantes sobre un grupo de pobladores que se vieron obligados a emigrar a causa de las pésimas condiciones ambientales que imposibilitaban continuar con sus tradicionales métodos de producción.

Considero que este tipo de investigaciones son necesarias para poder plantear una respuesta fundamentada, y poder responder al tipo de rol que los gobiernos deben ejercer. Si ya no existe un acuerdo en cuanto al concepto de migrante climático, será aún más difícil poder conseguir las voluntades políticas que acepten brindarle una protección legal acorde.

\section{$3 \quad$ El migrante ambiental y su reconocimiento legal}

El cambio climático se nos presenta como la consecuencia ineludible de un proceso de desarrollo económico salvaje que no ha sabido medir todas las repercusiones de sus actos, a corto, mediano y largo plazo.

Nos encontramos hoy en día ante una enorme cantidad de evidencia científica, que nos indica que los cambios en los patrones del clima no pueden ser vistos exclusivamente bajo una óptica técnica-científica, los mismos requieren de la necesaria intervención del derecho y de las políticas públicas.

Lo ideal es que el avance en ambos aspectos vaya de la mano, a fin de tener posiciones programáticas claras que nos guíen al desarrollo de una agenda ambiental encaminada a obtener el tan deseado desarrollo sostenible definido en el Informe Brundtland de 1987.

Un desarrollo que satisfaga las necesidades del presente sin comprometer las necesidades de las generaciones futuras. Es decir, un desarrollo que incorpore de manera armónica los componentes ecológico, económico y social.

2Para mas información consultar el portal del proyecto http://www.each-for.eu/, y http://www.ccemaportal.org/article/read/each-for-project-publications. 
Invest. pens. crit. (ISSN 1812-3864)

Vol. 5, No. 1, enero-abril 2017

pp. 69-76

Nuestro interés en el argumento nace de experiencias previas, adquiridas con el estudio de las migraciones producto de factores económicos, políticos, sociales o familiares en el flujo de migrantes entre África y Europa. Presentándose un análisis de las causas, en ocasiones de manera bastante sectorizada, sin interés en una posible correlación del factor ambiental con un desplazamiento por un desastre natural puntual. Esta es una de las primeras dificultades en este tipo de estudio, e inclusive es definido por algunos como el principal motivo para su falta de definición, pues es muy difícil aislar los factores medioambientales del resto de elementos [5].

Para Morton y otros, el cambio climático afectará significativamente a las migraciones de tres formas diferentes: "la primera de ellas, a partir de la aridez de zonas agrícolas que reducirá su potencial económico y mermarán servicios naturales como agua potable y suelo fértil; una segunda modalidad, mediante inundaciones que provocarán desplazamientos masivos; y como última categoría, aquellos que serán desplazados por el aumento del nivel del mar que destruirán zonas productivas cercanas a la costa y destruirán el hábitat de millones de personas"[5]. Definición que encierra un amplio espectro de personas que clasificarían bajo la denominación como migrantes climáticos.

Es importante destacar que a nivel internacional existen experiencias documentadas e inclusive un antecedente digno de mención como es el caso Ioane Teitiota contra el Ministerio de Negocios, Innovación y Empleo.

El señor Teitiota es un ciudadano de la República de Kiribati, una pequeña isla en el Océano Pacífico, quien pidió al gobierno de Nueva Zelanda permanecer en dicho país, bajo la figura de refugiado climático. De hecho la amenaza climática no es probable sino más bien real en Kiribati, uno de los países catalogados como altamente vulnerables a los efectos del cambio climático.

La Corte Suprema de Nueva Zelanda en un fallo poco extenso (tan solo 6 páginas) logra llamar la atención de la comunidad internacional. ${ }^{3}$ Si bien el fallo niega al señor Teitiota su condición de refugiado climático, aclara que dicha decisión no significa que la degradación ambiental producto de cambios en el clima no pueda crear un camino legal para su reconocimiento en la Convención sobre el Estatuto de los Refugiados de 1951 [6].

Aunque para muchos este fallo sea desalentador, pienso que ha abierto el camino a un debate que

${ }^{3}$ Fallo de la Corte Suprema de Justicia de Nueva Zelanda SC7/2015, entre Ioane Teitiota vs el Ministerio de Negocios, Innovación y Empleo.[8] 
Invest. pens. crit. (ISSN 1812-3864)

Vol. 5, No. 1, enero-abril 2017

pp. $69-76$

podría provocar interesantes cambios legales en el orden jurídico internacional. El grupo de los Verdes en el Parlamento Europeo pone como ejemplo para el resto de los países miembros de la Unión

Europea a Suecia y Finlandia, que al menos tienen mecanismos de protección a este grupo de migrantes, al menos teóricamente [7]. Inclusive en estos casos, el mayor obstáculo radica en la respuesta política que ha sido dada a este hecho.

Actualmente, existen políticas públicas para los migrantes, aterrizando en estudios de carácter psicológico, social, económico y político que analizan sus causas; pero no se toma en cuenta si ese flujo de personas por aparentes razones económicas nace del cambio en las condiciones climáticas del lugar. El hecho de tener que buscar nuevas formas de sustento que supere el tradicional desarrollo económico debe considerar el drástico cambio climático como una causa de origen.

\section{$4 \quad$ Conclusiones}

El reconocimiento legal o no del migrante ambiental o climático debe ser el primer paso para poder generar una política migratoria consciente que existen otros factores causantes de la migración que merecen ser estudiados.

Si bien es probable que introducir esta nueva causal en el Convenio sobre refugiados de 1951 sea una labor que requerirá de muchos años, es importante que exista una protección legal al respecto, que muy bien puede ser abordada en un instrumento jurídico a parte.

Además creemos que la temática debe ser objeto de estudio y consideración en las Conferencias de las Partes que se realizan anualmente, como mecanismo de presión a los gobernantes que deben tomarse acciones al respecto, desde la conciencia que es un fenómeno existente, sin esperar enfrentarnos a los pronósticos realizados por ACNUR o el prof. Myers.

En el extenso proceso de crear políticas públicas que incluyan esta nueva categoría de migrantes, debemos partir del primer paso, es decir, buscar el reconocimiento de los migrantes ambientales y a partir de ello, crear los planes de adaptación donde se busque reducir la vulnerabilidad y crear ambientes resilientes a los efectos que los cambios ambientales han ido provocando.

A nivel internacional, vemos el rico debate existente sobre la presencia de un nuevo tipo de desplazamiento voluntario y forzado, que no tiene un reconocimiento legal y por ende un tratamiento jurídico 
Invest. pens. crit. (ISSN 1812-3864)

Vol. 5, No. 1, enero-abril 2017

pp. 69-76

uniforme. A nivel nacional, no hay una investigación relacionada con este binomio ambiente-migraciones, pero sería interesante iniciar el debate al respecto tomando como referencia algunos desplazamientos a lo interno de la República de Panamá, donde se ha verificado un deterioro ambiental.

\section{$5 \quad$ Referencias}

1. BROWN, O. El baile de las cifras. Revista Migraciones Forzadas. 2008, no. 31, pp.8-9.

2. EGEA, C. y J. SOLEDAD. Los Desplazados Ambientales, más allá del cambio climático. Un debate abierto. Cuadernos Geográficos. 2011, no. 49, pp. 201-215.

3. OIM. Sesión 94․ Documento MC/INF/288 "Discussion Note: Migration and the Environment".http://www.iom.int/jahia/webdav/shared/shared/mainsite/about_iom/en/coun cil/94/MC_INF_288.pdf

4. GRAY, C.. Environmental refugees or economic migrants? Population Reference Bureau, enero 2010. http://www.prb.org/Publications/Articles/2010/environmentalmigrants.aspx

5. MORTON, A., P. BONCOUR y F. LACZKO, F. Seguridad humana y desafíos políticos. Revista Migraciones Forzadas. 2008, No. 31, p.5.

6. NACIONES UNIDAS. Convención sobre el Estatuto de los Refugiados. Adoptada en Ginebra, Suiza, el 28 de julio de 1951 por la Conferencia de Plenipotenciarios sobre el Estatuto de los Refugiados y de los Apátridas (Naciones Unidas), convocada por la Asamblea General en su resolución 429 (V), del 14 de diciembre de 1950. Entrada en vigor: 22 de abril de 1954, de conformidad con el artículo 43 Serie Tratados de Naciones Unidas, No 2545, Vol. 189, p. 137.

7. FLAUTRE, H., J. LAMBERT, S. KELLER y B. LOCHBIHLER. Position paper: Climate change, refugees and migration. Los verdes en el parlamento europeo. 2013.

8. Fallo de la Corte Suprema de Justicia de Nueva Zelanda SC7/2015, entre Ioane Teitiota vs el Ministerio de Negocios, Innovación y Empleo.

9. ANAM. Informe del Estado del Ambiente GEO. Panamá, 2004.

10. ANAM. Informe GEO 2014. Panamá, 2014.

11. ANAM. Atlas de las tierras secas y degradadas de Panamá, 2009.

12. ANAM. Primera Comunicación Nacional ante la Convención Marco de las Naciones Unidas sobre el Cambio Climático. Panamá, 2000.

13. ANAM. Segunda Comunicación Nacional ante la Convención Marco de las Naciones Unidas sobre el Cambio Climático. 2a ed. Panamá: Autoridad Nacional del Ambiente, 2011.

14. BLANCO, P. y M. SAHUQUILLO. Los refugiados climáticos no tienen dónde buscar asilo. El País, en El País, 15 de diciembre de 2015. http://internacional.elpais.com/internacional/2015/12/14/actualidad/1450123647_559457.html

15. CATHALAC. Contaminación por atrazina, río La Villa, Azuero-Panamá. Julio 12, 2014. 
Invest. pens. crit. (ISSN 1812-3864)

Vol. 5, No. 1, enero-abril 2017

pp. $69-76$

http://www.servir.net/servir-en-accion/analisis-ambientales/553-contaminacion-por-atrazinario-la-villa-azuero-panama.html

16. Colaboradores de Enciclopedia. Península de Azuero (Panamá). En: Enciclopedia Libre Universal en español. http://enciclopedia.us.es/index.php?title $=$ Especial:Citar\&page $=$ Pen $\%$ C3\%ADnsula_de_Azuero_\%28Panam\%C3\%A1\%29\&id $=579490$

17. ESPÓSITO, C, y A. TORRES. Cambio climático y derechos humanos: el desafío de los nuevos refugiados, pp. 26. http://www.palermo.edu/derecho/revistaderechoambiental/pdfs/REV12012-Derecho_Ambiental_01.pdf

18. GLAHN, B,. Climate refugees'? Addressing the international legal gaps - Part II. International Bar Association. http://www.ibanet.org/Article/Detail.aspx?ArticleUid=3E9DB1B0-659E-432B8EB9-C9AEEA53E4F6 (03.08.2009).

19. Glosario de la Organización Internacional para las Migraciones (OIM), el cual puede ser consultado en http://www.iom.int/es/los-terminos-clave-de-migracion

20. INEC. Distribución Territorial y Migración Interna en Panamá: Censo 2010. Panamá: Contraloría General de la República, 2014.

21. Instituto Interamericano de Cooperación para la Agricultura (IICA). http://www.iica.ac.cr/Esp/prensa/paginas/comunicadoprensav1.aspx?cp=174

22. MARCELLESI, F. y R. MARTÍNDEZ. Reconocer y proteger a los refugiados climáticos. El País. 27 de octubre de 2015 http://internacional.elpais.com/internacional/2015/10/26/actualidad/1445872107_049759

23. Resolución de Gabinete No.63 de 7 de mayo de 2013, que declara el Estado de Emergencia en la denominada región del Arco Seco, que abarca las provincias de Coclé, Herrera, Los Santos y Veraguas. Gaceta Oficial No.27282-A de 8 de mayo de 2013.

24. United Nations Regional Information Centre for Western Europe. "The invisible climate refugees". http://www.unric.org/en/latest-un-buzz/28883-the-invisible-climate-refugees (02.12.2013).

25. MARTÍ, R. La diferencia entre 250 y 1.000 millones de refugiados climáticos depende de un grado (Entrevista con Florent Marcellesi), 03.12.2015. http://www.playgroundmag.net/articulos/entrevistas/diferencia-millones-refugiados-climaticos-depende_0_1656434345.html

26. HOFFMANN, D, No hay migrantes climáticos (Entrevista a Raoul Kaenzig) 18.02.2013. http://www.cambioclimatico-bolivia.org/pdf/cc-20130218-_quot_No_hay_migrantes_clim_aacute_ticos_quot_Entrevista_al_investigador_suizo_Raoul_Kaenzig.pdf 